\title{
Les participiales absolues sont-elles des subordonnées circonstancielles? A propos de la sous-spécification des rapports syntaxo-sémantiques
}

\author{
Eva Havu', et Michel Pierrard ${ }^{2}$ \\ ${ }^{1}$ Université de Helsinki, Finlande \\ ${ }^{2}$ Vrije Universiteit Brussel, Belgique \\ eva.havu@helsinki.fi
}

\begin{abstract}
Résumé. Les constructions participiales absolues (CPA) sont généralement réparties en deux types de constructions, selon (a) qu'elles sont interprétées comme une structure à prédication seconde, " liée à un argument dans la prédication principale par une relation de possession » et qui « dénote une manière " (Mouret, 2011 : 51) ou (b) qu'elles peuvent être paraphrasées par une subordonnée circonstancielle. Nous examinerons les participiales absolues de type (b) en confrontant leurs propriétés avec celles des subordonnées circonstancielles (SC) sur trois plans : 1. les contraintes sémantiques pesant sur l'interprétation des prédications connectées ; 2. le mode de connexion entre les prédications ; 3 . la caractérisation des rapports syntaxiques liant les prédications. Notre contribution se donne pour objectif de préciser les rapports syntaxo-sémantiques instaurés par les deux constructions (CPA et SC).
\end{abstract}

\begin{abstract}
Are absolute participial constructions adverbial subordinate clauses? On the underspecification of semantico-syntactic relations. Generally absolute participial constructions (APC) are considered to represent two distinct types according to their interpretation either as (a) a secondary predication expressing a possessive relation with an argument in the main clause and denoting manner (Mouret, 2011: 51) or as (b) parphrasing an adverbial subordinate clause. We examine absolute participial constructions of type (b) and compare their properties with those of adverbial subrodinate clauses (AS) by discussing three characteristics: 1 . semantic constaints in the interpretation of the connected predications; 2. types of connection between the predications; 3. the characteristics of syntactic relations between the predications. Our objective is to specify the semantico-syntactic relations implemented by the two constructions (APC and AS).
\end{abstract}

Les participiales absolues sont généralement réparties en deux types de constructions (e.a. Hanon 1989, Mouret, 2011, Havu \& Pierrard, 2016a ${ }^{1}$ ), selon (a) qu'elles sont interprétées comme une structure à prédication seconde, « liée à un argument dans la prédication principale par une relation de possession » et qui « dénote une manière » (Mouret, $2011: 51$ ) (1a-2a) ou (b) qu'elles peuvent être paraphrasées par une subordonnée circonstancielle (1b-2b) :

(1a) Marie est assise, les yeux fermés. [Marie est assise et elle a les yeux fermés]

(1b) La porte fermée, Marie s'en alla.

(1b') Comme la porte était fermée / après avoir fermé la porte, Marie s'en alla.

(2a) A... n'a pas bronché depuis sa découverte : très droite sur sa chaise, les deux mains reposant à plat sur la nappe de chaque côté de son assiette. (Robbe-Grillet : 62 ; apud Mouret, ex. 11a).

(2b) L'avion ayant atterri avec une heure de retard, elle a pris un taxi.

(2b') Après que/ comme/ parce que l'avion a atterri avec une heure de retard, elle a pris un taxi.

La construction participiale absolue (CPA) dans $1 b-2 b$, qui fait l'objet de la présente étude, se caractérise par plusieurs traits : (a) elle constitue une prédication complète, l'union d'un thème et d'un prédicat, (« [...] 
les formes qui dénotent une circonstance $[\ldots]$ seront analysées comme des phrases à part entière » (Mouret, $2011: 51)$ ) ; (b) elle exprime une circonstance en mettant « en relation de cause à effet ou en relation de temporalité deux prédications » (Hanon, $1989: 63$; cf. aussi Mouret, $2011: 51$ ). La CPA dans ce cas est analysée comme une « construction absolue circonstancielle » (Mouret, 2011 : 52); selon Hanon (1989: 63-64), elle est « issue d'une subordonnée circonstancielle » ou encore, elle « joue le même rôle que la proposition adverbiale » (Grevisse \& Goosse, $2008: 1478\left(\$ 1134, \mathrm{c}, 2^{\circ}\right)$. Peut-on dès lors assimiler fonctionnellement la CPA aux adjoints circonstanciels et plus spécifiquement aux subordonnées adverbiales circonstancielles au moyen desquelles elle est souvent glosée (1b'-2b') ?

La problématique abordée ici concerne un questionnement plus large impliquant toute une série de constructions adjectivales ou participiales adjointes qui permettent également une glose circonstancielle (cf. Goes 2008, Riegel 1991 et Muller 2000) :

(3a) Paul est rentré ivre. [Paul est rentré alors qu'il était ivre.]

(3b) Paul boit son café chaud. [Paul boit son café quand il est chaud.]

Ces adjectifs ou participes, qui présentent à l'origine un ancrage nominal (participant orientation), tendraient dans certains emplois à porter sur le prédicat (event-orientation) et à glisser en conséquence vers un fonctionnement de type adverbial (cf. Himmelmann \& Schulze-Berndt, 2005). Bref, comment expliquer ce sens circonstanciel que prend la CPA, découle-t-il du rôle fonctionnel d'adjoint circonstanciel qu'elle est amenée à remplir à travers la connexion de prédications ou constitue-t-elle tout simplement une subordonnée circonstancielle à verbe non fini ?

A partir d'un corpus de $250^{2}$ occurrences de CPA incluant aussi bien des participes présents que des participes passés, nous confronterons les propriétés des CPA avec celles des subordonnées circonstancielles (SC) sur les trois plans suivants :

(1) les contraintes sémantiques pesant sur l'interprétation des prédications connectées ;

(2) le mode de connexion entre les prédications ;

(3) la caractérisation des rapports syntaxiques liant les prédications.

Notre contribution se donne pour objectif de préciser les rapports syntaxo-sémantiques instaurés par les deux constructions (CPA et SC).

\section{Contraintes interprétatives sur les prédications connectées}

Sur un plan sémantique, une première différence qui s'impose de prime abord entre les SC et les CPA semble être le degré de précision sémantique, puisque les énoncés ( $1 \mathrm{~b}-2 \mathrm{~b})$ permettent plusieurs lectures (cf. $\left.1 b^{\prime}-2 b^{\prime}\right)$. Examinons de plus près les contraintes interprétatives qui pèsent sur chacun des types.

\subsection{Mode d'élaboration de la lecture circonstancielle}

Un test demandant à trente étudiants universitaires francophones de paraphraser 20 constructions adjointes participiales ou adjectivales (Havu, 2004) a abouti au résultat que seuls 5 des 20 exemples ont été interprétés d'une manière tout à fait univoque (4a), que ce soit par une interprétation argumentative ou temporelle (antériorité, simultanéité, postériorité). Dans 5 exemples, l'interprétation argumentative ou temporelle du participe variait beaucoup (4c), tandis que dans les 10 derniers exemples, on trouvait de la variation soit au niveau de l'interprétation sémantique, soit au niveau de l'interprétation temporelle (4b) :

(4a) Peu à peu l'identité de MG «s'efface», blessée que «cet homme voie en elle un ventre et non une femme». [parce qu'elle était blessée que...]

(4b) Alliés comme jamais pour combattre le terrorisme, les deux chefs d'État [Vladimir Poutine, George W. Bush] vont-ils définir d'une même voix l'après-talibans ? [Bien qu'ils soient / Comme ils sont...] 
(4c) Réélu après une carrière politique semée de revers et d'amitiés trahies, le président s'est forgé un destin d'exception (cause, temps, concession, vide) [Comme le président est / a été réélu // Bien que le président soit / ait été réélu // Après avoir été réelu //Le président a été réélu et il s'est forgé....]

Là où le connecteur explicite d'emblée l'interprétation chez les SC, celle-ci est liée dans le cas des CPA au sens véhiculé par les deux prédications, parfois à leur position respective et même au contexte discursif plus général :

(5a) Quelques mois plus tard, son immunité ayant été levée, il a fui en France pour échapper à une nouvelle arrestation. (Le Monde 12-13.6.2005/21) [lorsque son immunité a été levée/parce que son immunité avait été levée...]

(5a') Quelques mois plus tard, il a fui en France pour échapper à une nouvelle arrestation, son immunité ayant été levée. [??lorsque son immunité a été levée/parce que son immunité avait été levée...]

(5b) Les bijoux vendus, elle est repartie avec 500 euros. TF1 - Sept à huit 06/05/2012 - 18h00 [Après que /comme les bijoux ont été vendus...]

(5b') Elle est repartie avec 500 euros, les bijoux vendus. [Après que / ??comme les bijoux ont été vendus...]

Dans le cas des exemples (5a-b), le contexte instille une interprétation temporelle ou causale mais ne permet pas de trancher entre les deux, alors que le connecteur la sélectionne explicitement dans le cas des SC. Le changement de position de la participiale semble favoriser une interprétation univoque, causale dans (5a') et temporelle dans (5b').

L'interprétation des conjonctions subordonnantes dans les SC n'est toutefois pas toujours univoque non plus (Riegel et al., 2009: 848), en particulier dans le cas des conjonctions « primaires » quand, comme et $s i$ : certaines conjonctions considérées généralement comme temporelles $(6 \mathrm{a}, \mathrm{d})$ se prêtent également à d'autres interprétations : comme peut être causal (6b) et comparatif (6c) et quand peut exprimer une opposition (6e) et une hypothèse (6f) :

(6a) Comme nous approchions de la ville, il s'est mis à pleuvoir

(6b) Comme j'étais pressé, j'ai voyagé en avion.

(6c) Il ment comme il respire.

(6d) Quand [lorsque] il viendra, je lui expliquerai tout.

(6e) Tu t'es subordonné, quand [alors que] tu es fait pour ordonner. (Balzac)

(6f) Un ancien intellectuel, quand [même si] il serait devenu maçon, [...] est toujours un aristo. (Péguy)

Bref, l'interprétation de la prédication régie est explicitement orientée dans le cas des SC, alors que cette orientation est implicite dans le cas de la CPA. En conséquence, le mode d'élaboration de la lecture circonstancielle est plus contraint mais aussi plus précis dans le premier cas, moins contraint mais aussi plus imprécis dans le second cas.

\subsection{Limites de l'interprétation circonstancielle des CPA}

À première vue, la CPA ne permet pas toutes les interprétations circonstancielles. Une première limite concerne la temporalité. Si l'antériorité ou la simultanéité d'un événement par rapport à celui exprimé par la régissante peut être marquée par les formes participiales (7a-b et 7a'-b'), ce n'est pas le cas de la postériorité. Cependant, l'utilisation d'autres marqueurs adverbiaux ou aspectuels permet d'orienter la CPA vers l'expression de la postériorité (7c') :

(7a) Ses parents sont arrivés après que l'appartement avait été nettoyé/ après que Paul avait nettoyé l'appartement.

(7a') L'appartement (ayant été) nettoyé, ses parents sont arrivés/ Paul ayant nettoyé l'appartement, ses parents sont arrivés

(7b) Ses parents arrivent pendant que Paul nettoie l'appartement/ l'appartement est nettoyé.

(7b') Paul nettoyant l'appartement, ses parents arrivent. 
(7c) Paul nettoie l'appartement/ L'appartement est nettoyé avant que ses parents n'arrivent.

(7c') Ses parents arrivant bientôt/ ses parents devant arriver, Paul nettoie l'appartement ${ }^{3}$.

Le corpus contient effectivement des exemples exprimant l'antériorité ( $7 \mathrm{~d}:$ « après que ») et la simultanéité (7e: «tandis que »), beaucoup plus rarement et dans ce cas avec un marqueur adverbial (alors), la postériorité (7f : « avant que ») :

(7d) «[...]. En menant une politique de réformes socio-économiques, nous voulons éveiller l'appétit des francophones. " Sous-entendu : Des réformes libérales imposées à une Wallonie considérée comme socialiste ou communiste, la demande de réformes arrivera côté wallon. Pour Bart De Wever, cette stratégie est nécessaire parce « qu'on peut retourner le problème dans tous les sens, nous aurons besoin d'une majorité des deux tiers, dans chaque groupe linguistique. » (Le Soir, 04.05.2017/5) [une fois que / après que des réformes ont été imposées...]

(7e) La ville bruisse de sirènes. Des quatre coins de la ville, les voitures de pompiers appelées en renfort convergent vers le lieu du sinistre. Dans la rue Neuve et à l'arrière du magasin, les clients les plus chanceux s'échappent. Tous les étages envahis d'une fumée dense, la panique est à son comble. Le feu gagne l'ensemble du magasin, se propageant par les faux plafonds. Des dalles enflammées tombent sur les articles textiles, amplifiant encore la force du sinistre. (Le Soir, 20.05.2017/12) [tandis que tous les étages sont envahis ....]

(7f) C'est lui [intermédiaire], selon nos informations, qui renoue, fin mars, le contact avec Philippe Brett, celui-ci affirmant alors à des journalistes qu'il informe le Quai d'Orsay et Libération de ses discussions. ( Le Monde, 14.6.05/ 4)

Une deuxième limite envisageable concerne l'expression de la gamme totale des diverses facettes de la lecture circonstancielle. Les lectures causale (8a) causale renversée (8b) et temporelle (8c) sont, comme les exemples précédents le démontraient déjà, les deux lectures les plus courantes :

(8a) La minuterie étant hors service, Baumgartner grimpe aveuglément jusqu'au dernier étage. (Echenoz, p. 87) [comme la minuterie est hors service,...]

(8b) La première raison est le plan de bataille. Alors que les quartiers situés sur la rive orientale du Tigre furent presque tous conquis par les bataillons du Service de contre-terrorisme (CTS), l'unité de forces spéciales connue sous le nom de «Division d'Or », les opérations menées sur l'autre rive sont partagées entre trois forces qui avancent conjointement vers le nord : le CTS, la Police fédérale et la Division de réaction d'urgence (ERD), ces deux dernières dépendant du ministère de l'intérieur. Cet ordre de bataille amène davantage de confusion, les cultures et les capacités militaires s'avérant très inégales. (Le Monde, 11.3.2017, http://www.lemonde.fr/proche-orient/article/2017/03/11/a-mossoulvive-resistance-de-l-ei-sur-la-rive-occidentale-du tigre 5092952 3218.html?xtmc $=$ s averant\&xtcr=1_ [Cet ordre amène davantage de confusion, étant donné que les cultures et les capacités...; Comme les cultures et les capacités..., cet ordre amène...]

(8c) Le moment venu, je poursuivrai tous ceux qui sont à l'origine de cette affaire. (Journal Télévisé :

Lalibre.be en ligne 06.04.17 URL : http:/www.lalibre.be/video/fillon-accuse-hollande-le-moment-venu -jepoursuivrai-tous-ceux-qui-sont-a-l-origine-de-cette-affaire-58e613e7cd70e80512b0e219_[quand le moment sera venu,...]

Une lecture concessive (8d) ou oppositive (8e) n'est pas impossible et Müller-Lancé (1998) fournit même un exemple qui suggérerait une lecture conditionnelle $(8 \mathrm{f})$, mais on arrive ici à des interprétations de plus en plus subjectives :

(8d) Ce n'est ici un secret pour personne - le négociateur de l'ONU ne pouvant évidemment l'avouer - que l'ancien « maître de Kaboul » était toujours réfugié lundi soir au siège local des Nations Unies. (Müller-Lancé 1998 : 270-271) [même si le négociateur de l'ONU ne pouvait pas l'avouer...]

(8e) Le ministre des finances britannique, Gordon Brown, a rejeté cette suggestion, M. Blair se montrant plus flou. (Le Monde 14.6.2005/8) [... tandis que M. Blair se montrait plus flou]

(8f) Il faut montrer des résultats pour obtenir le soutien des sponsors, ce qui implique d'avancer les fonds nécessaires afin d'acquérir un matériel performant et se payer les premiers voyages. Cette étape franchie, il faut assurer pour garder ces éventuels sponsors et se garantir les moyens de gagner un titre. [Quand / ? si cette étape est franchie, il faut...]

Il en va de même des interprétations exprimant une conséquence $(8 \mathrm{~g})$. Celle-ci est souvent imposée au moyen d'un indice complémentaire renforçant cette lecture $(8 \mathrm{~h}$ : ainsi) : 
(8g) Semaine après semaine, une salve régulière d'accusations se fait alors entendre à Washington, la liste des erreurs prêtées au candidat socialiste ne cessant de s'allonger et de se diversifier. Après sa victoire dans le New Hampshire, le 9 février, le Washington Post le qualifie, au même titre que M. Trump, de « dirigeant intolérable » qui ne propose que des solutions «simplistes ». (Le Monde Diplomatique, déc. 2016 : 18) [...de sorte que la liste des erreurs ne cesse d'allonger...]

(8h) Vote historique à la quasi-unanimité : 2896 voix pour, 2 abstentions et zéro contre. Le Parlement chinois adoptant ainsi une loi qui prévoit le recours à la force si Taiwan déclare son indépendance. La Chine n'a jamais renoncé à Taiwan avec ses 23 millions d'habitants. (FR2, JT de 20h, 14.03.05) [..., de sorte que le Parlement chinois adopte une loi...]

En résumé, si les « scénarios interprétatifs » standards des CPA sont ceux menant à une lecture temporelle (simultanéité et antériorité) ou causale, il n'y a pourtant pas de limites interprétatives dans la mesure où tous les sens circonstanciels semblent être possibles. Cependant, de telles lectures «non standard» demandent alors souvent des indices spécifiques : adverbes ou autres marqueurs, aspect lexical, verbes modaux, contexte discursif orienté.

\subsection{Interprétations non circonstancielles des CPA}

Les CPA peuvent aussi mener à une lecture non circonstancielle, une lecture qui ne sera donc pas paraphrasée par une SC. Elles apparaissent alors souvent en postposition, dans un contexte d' «enchaînement de prédications » (phénomène de " clause chaining », cf. Givon 1990 : 864) et participent à l'expression d'une successivité discursive, d'un enchaînement d'événements (9a) ou encore sont interprétées comme des ajouts descriptifs (9b). Pour accentuer l'effet de successivité discursive qu'elles instillent, les CPA sont souvent accompagnées d'une expression temporelle (9a : alors; 9c : jusqu'à 22h30):

(9a) $[\ldots]$ : un village vidé de ses habitants, puis rénové ou même partiellement reconstruit à l'ancienne, certains étant alors invités à revenir pour animer ce «village touristique » à la vie artificielle. (Le Monde diplomatique, novembre 2015/5)

(9b) Elle [évolution] fait apparaître une intégration sans précédent des satellites aux autres sources de reconnaissance, de communication et de commandement - l'architecture d'ensemble dépendant de façon critique de télécommunications sûres et à haute capacité via satellites. (Le Monde, 14.6.2005/1)

(9c) Sur 57429 inscrits sur la liste électorale à Montréal, 22234 ont voté le 22 avril mais nombreux ont été ceux qui n'ont pas pu ou voulu attendre plusieurs heures dans la rue, par un temps froid, avant de pouvoir atteindre l'isoloir. De sérieux efforts avaient pourtant été faits par les autorités consulaires. Tous les électeurs arrivés avant 20 heures ont ainsi pu voter, les opérations électorales s'étant poursuivies jusqu'à $22 \mathrm{~h} 30$. Le Monde, 3.5.2017, http://www.lemonde.fr/election-presidentielle-2017/article/2017/05/03/a-montreal-le-consulat-veut-corriger-les-ratesdu-premier-tour_5121599_4854003.html?xtmc=s_etant\&xtcr=2

D'autre part, les CPA ont souvent aussi un ancrage nominal (participant orientation) et non pas verbal, ce qui les différencie à nouveau des $\mathrm{SC}$ :

(9d) Cette agitation pacifique a déclenché en retour la violence des islamistes, elle-même durement réprimée. (Le Monde Diplomatique, déc. $2016: 8$ )

(9e) James Comey n'ayant pas obéi, le président l'aurait limogé, (« des accusations balayées dans une conférence de presse ». France 2, JT 20H, 19/05/2017 (22:22-24), https:/www.youtube.com/watch?v=GE7RI05CuiU)

\subsection{Différences dans les contraintes interprétatives}

Les contraintes interprétatives sur les prédications connectées sont très différentes dans le cas des SC et des CPA. En premier lieu, le mode d'élaboration de la lecture circonstancielle est plus contraint mais aussi plus précis dans le premier cas, moins contraint mais aussi plus imprécis dans le second cas. En deuxième lieu, les interprétations circonstancielles ne sont pas les seules lectures générées par les CPA, alors qu'elles sont exclusives dans le cas des SC. 


\section{Le mode de connexion entre les prédications}

Sur un plan purement formel, la différence la plus saillante entre les deux constructions consiste en la présence (1b'-2b') ou l'absence (1b-2b) d'un connecteur interpropositionnel entre les prédications (Hobaek Haff, 2010 : 208-209). Ce connecteur est généralement convoqué pour identifier une liaison entre prédications ( «Les subordonnées sont, en principe, reconnaissables à la présence d'un terme introductif » (Arrivé, Gadet \& Galmiche, $1986: 640$ ) ; « There is likely to be a grammatical marker attached to one of the clauses (in most cases, not to both) indicating the type of linking involved » (Dixon, $2011: 3$ )).

La connexion de prédications implique-t-elle nécessairement la présence d'une marque syndétique, d'un élément liant et marquant explicitement le rapport syntaxo-sémantique entre deux prédications ? Dans une approche typologique de la jonction de propositions, Lehmann $(1988: 210)$ identifie divers « struments connectifs » (il parle de « connective device »). Pour ce qui concerne la subordination (Ibid. : 212-213), il répertorie comme "strument connectif » non seulement les mots subordonnants (tels les conjonctions), mais aussi des séquences anaphoriques (au moment où, de manière que). De plus, dans la perspective d'une approche typologique du participe (Haspelmath 2010), Cristofaro (2003) ou Dixon (2011) soulignent que la subordination peut, elle aussi, adopter un mode asyndétique par l'emploi de formes non finies. L'absence de marqueur syndétique n'exclut donc pas l'instauration d'une connexion entre prédications.

\subsection{Modes de connexion opérant dans la CPA}

Deux types de phénomènes permettant d'établir une connexion entre deux prédications dans le cas des CPA (cf. Havu \& Pierrard, 2015, 2016a).

2.1.1. La forme non finie du verbe d'une des deux prédications instaure une dépendance TAM du participe par rapport au verbe fini et donc de la participiale par rapport à la proposition régissante. La CPA (10a) partage d'ailleurs cette caractéristique avec les participiales adjointes (10b) :

(10a) L'hiver venu, les squelettes de ces plantes forment une structure délicate dans de riches déclinaisons de brun. (Fin du paragraphe) LeMonde.fr en ligne $\mathrm{M}$ le Magasin du Monde 13.01.16, http://www.lemonde.fr $/ \mathrm{m}-$ perso/article/2016/01/13/la-seconde-vie-des-fleurs-1-hiver_4843141_4497916.html

(10b) Marginalisés pour s'être compromis avec le Pakistan lors de la guerre de libération en 1971, les islamistes bangladais retrouvent leur influence : (Le Monde Diplomatique, déc. 2016 : 8).

2.1.2. Cependant un autre phénomène instaure une connexion entre les deux prédications. Il s'agit d'un lien référentiel entre le thème de la $\mathrm{CPA}$ et un $\mathrm{N}$ de la régissante. Cette dépendance nominale peut se concrétiser sous différentes formes (co-référence, anaphore associative, reprise pronominale d'un $\mathrm{N}$ ) :

A un premier niveau, le lien prend la forme de la coréférence entre le $\mathrm{N}$ support du co-prédicat et un $\mathrm{N}$ de la prédication régissante : cela va de la coréférence, l'utilisation de $\mathrm{N}$ différents renvoyant au même référent (11a ; cf. pape / Jean-Paul II) à l'anaphore associative entre deux N sous la forme par exemple d'un rapport partie/ tout (11b ; réfrigérateur/ freezer) :

(11a) Dans l'actualité de ce dimanche : l'apparition du pape à la fenêtre de l'hôpital, Jean-Paul II se recueillant ce matin au moment de la prière de l'Angélus. (TF1, JT de 13h, 27.02.05)

(11b) Le réfrigérateur étant très peu utilisé, un iceberg naturel envahissait le freezer que Ferrer, quand cet iceberg virait à la banquise, dégivrait tous les ans à l'aide d'un sèche-cheveux et d'un couteau à pain. (Echenoz, p. 17)

A un stade ultérieur, la coréférence se matérialise lexicalement sous la forme d'une co-lexicalisation, le même terme apparaissant dans la prédication régissante et comme support du prédicat participial. Il y a donc ici sélection du lexème précis autour duquel s'agence le lien entre les prédications, mais ce lien ne s'inscrit pas encore dans une configuration grammaticale :

(11c) On sentira la différence au niveau des températures puisque nous allons perdre de 10 à 12 degrés, les températures demain variant entre 15 et 21 degrés. RTBF - Journal télévisé de 19:30 h (17/5/2017) 
La reprise partielle/ restreinte du $\mathrm{N}$, de type distributif ou quantifiant, dans une des deux prédications souligne un début d'inscription du rapport dans une configuration grammaticale, puisqu'il n'y a plus d'équivalence lexicale et que l'interprétation du thème de la CPA dépend d'un processus de calcul anaphorique :

(11d) Il n'en s'agit pas moins de deux courants distincts, le premier étant plutôt situé à droite et le second reflétant la domination idéologique persistante du marxisme au sein d'une partie de la gauche française. (Le Figaro 7.12.2004/12)

A partir de ce type d'énoncés, la matérialisation du lien interprédicationnel implique l'émergence d'un mode de liaison spécifique, de type anaphorique, entre les prédications. Le N support est repris par une proforme nominale sémantiquement totalement dépendante du N dans la CPA (par exemple 11e : Philippe Brett, celui-ci ; 11f: Michelle Bachelet, elle aussi) :

(11e) C'est lui [intermédiaire], selon nos informations, qui renoue, fin mars, le contact avec Philippe Brett, celui-ci affirmant alors à des journalistes qu'il informe le Quai d'Orsay et Libération de ses discussions. (Le Monde 14.6.2005/4)

(11f) Il y a une autorité morale chez cette femme qui peut éventuellement s'étendre au Chili de Michelle Bachelet, elle aussi éduquée en Allemagne de l'Est. (Le Monde 22-23.1.2006/16)

\subsection{Le rôle spécifique du connecteur interpropositionnel ?}

Si ce qui différencie la CPA de la SC n'est pas le fait d'instaurer une connexion entre prédications malgré l'absence d'un connecteur interpropositionnel, quel est alors l'apport de ce dernier? Le connecteur interpropositionnel intervient sur deux plans (cf Havu \& Pierrard 2016c) :

2.2.1. En premier lieu, le connecteur fournit des informations sur le mode d'insertion de la prédication régie dans la régissante :

(12a) Épuisés par une conquête du pouvoir qui n'en finit pas, les proches de Marine Le Pen, eux-mêmes déstabilisés par le spectacle humiliant du débat d'entre-deux tours qui a opposé la benjamine de Jean-Marie Le Pen à Emmanuel Macron, se sont choisis un bouc émissaire en la personne de Florian Philippot. (Paris Match, 13-19.07.17, p.24)

(12a') [...], les proches de Marine Le Pen, qui eux-mêmes sont déstabilisés par le spectacle humiliant du débat d'entredeux tours $[\ldots]$, se sont choisis un bouc émissaire $[\ldots]$.

(12b) Pour le pouvoir royal, éleveurs de rennes et colons pouvaient se côtoyer sans se gêner. Mais subsister de la seule agriculture s'avérant impossible sous ces latitudes, les colons devaient chasser et pêcher... (Le Monde Diplomatique, déc. $2016: 11)$

(12b') Mais, comme subsister de la seule agriculture s'avérait impossible sous ces latitudes, les colons devaient chasser et pêcher...

Le connecteur explicite la manière dont la prédication régie est insérée dans la régissante : comme complément d'un $\mathrm{N}$ dans (12a'), comme complément circonstanciel dans (12b'). Cette information n'est pas fournie par la CPA correspondante (12a-b).

2.2.2. Par ailleurs, ce même connecteur fournit des informations sur la lecture sémantique du rapport connectif ( $\mathrm{cf} \S 1.1$.) :

(12c) Vendredi midi, le mariage signé entre leurs deux institutions, les recteurs de l'UCL et de Saint-Louis (USL-B) remettent d'emblée les pendules à l'heure. (Le Soir, 20.05.2017/10)

(12c') Vendredi midi, après que le mariage a été signé entre leurs deux institutions, les recteurs de l'UCL et de SaintLouis (USL-B) remettent d'emblée les pendules à l'heure.

(12d) L'année passée on a installé une fascine. Une fascine, c'est un peu un filtre, c'est une petite haie avec de la paille dedans. Et donc la fascine doit retenir la boue et laisser passer l'eau. Le problème c'est qu'après deux orages la boue continue à monter et pour finir la boue passe au-dessus de la fascine. Donc la fascine ayant montré ses limites, on doit chercher un complément. RTBF - Journal télévisé de 19:30 h (5/5/2017) - 21 :50

(12d') Comme la fascine a montré ses limites, on doit chercher un complément. 
Le connecteur impose explicitement une lecture temporelle dans (12c') et argumentative dans (12d'), contrairement aux CPA dans (12c-d), qui instillent une certaine ambigüité dans leurs lectures.

Cette interprétation ambiguë des CPA, faute de connecteur interpropositionnel, peut cependant être partiellement compensée par la flexion verbale (forme composée qui oriente nécessairement vers l'antériorité temporelle ou argumentative (causale) ; 12e), ou être levée par la présence d'indices de type adverbial (à peine, une fois...; 12f-g) ou encore par les propriétés aspectuelles lexicales des verbes (verbes téliques comme passer, terminer, $\ldots ; 12 \mathrm{~h})$ :

(12e) Karim C. prend la fuite et les deux hommes décident de suivre sa voiture. Intrigué par la plaque d'immatriculation qui lui semble fausse, le futur gardien de la paix alerte ses collègues. Karim C. ayant fait une nouvelle erreur de conduite, son véhicule finit dans le fossé. (Le Figaro, 21.4.2017, http://www.lefigaro.fr/actualitefrance/2017/04/21/01016-20170421 ARTFIG00078-des-2001-1-explosion-de-violence-de-karim-c-l-auteur-presumede-l-attaque-sur-les-champs-elysees.php)

(12f) Et il faut se souvenir que dans le cas de l'Allemagne, une fois le procès de Nuremberg terminé, les procureurs et les juges pouvaient faire leurs valises et rentrer chez eux, aux quatre coins de la planète, [...] (Le Monde 2223.1.2006/16)

(12g) Nous sommes devant la Chancellerie à Berlin, le siège du gouvernement allemand. Ces soldats qui se déploient à grand coup de musique militaire annoncent une visite importante, celle du nouveau président français, Emmanuel Macron. À peine le cortège official arrivé, Angela Merkel sort du bâtiment pour accueillir son visiteur. RTBF - Journal télévisé de 19:30 h (15/5/2017) - 5:29

(12h) La première surprise passée, il devint orange. (Nothomb, p. 141)

Si l'asyndèse n'élimine pas nécessairement le lien, elle a cependant un impact sur la (sous-) spécification de celui-ci : l'asyndèse tend à rendre le lien entre les prédications plus opaque, même si d'autres formes de liaison entre les prédications peuvent contrebalancer la sous-spécification et que le marquage de ce rapport peut se faire au moyen de divers indices autres que les « struments connectifs » classiques.

\subsection{Modes de connexion des CPA et des SC ?}

Les indices de connexion peuvent donc être de divers ordres et ne se limitent pas aux connecteurs. Cependant, l'apport de ces indices est fondamentalement opposé dans le cas des deux constructions qui nous occupent :

- dans le cas de la SC, la connexion est réalisée explicitement et directement par l'insertion d'un élément spécifique qui indique le point d'ancrage de la prédication régie, informe sur la lecture de la connexion entre les prédications et n'altère pas fondamentalement celles-ci ;

- pour ce qui concerne la CPA, la connexion est réalisée indirectement et implicitement par un manque, une absence affectant une des prédications et la rendant en conséquence dépendante de l'autre. La sousspécification de la prédication connectée déclenche la connexion mais rend celle-ci également par la même occasion sous-spécifiée.

\section{La caractérisation des liens syntaxiques entre les prédications}

Les contraintes interprétatives pesant sur les deux tours sont différentes et le mode de connexion est également radicalement distinct. Que révèlent ces divergences quant aux caractéristiques du rapport syntaxique liant les prédications dans les deux constructions?

\subsection{Différence des rapports syntaxiques avec la régissante}

La différence du rapport syntaxique liant les prédications apparaît à travers le comportement différent des SC (1b', 2b') et des CPA (1b, 2b) face au test du clivage :

(1b) La porte fermée, Marie s'en alla. 
(2b) L'avion ayant atterri avec une heure de retard, elle a pris un taxi.

(1b') Après avoir fermé la porte, Marie s'en alla.

(2b') Après quel parce que l'avion a atterri avec une heure de retard, elle a pris un taxi et est arrivée juste à temps pour sa réunion.

Le clivage est généralement autorisé dans le cas des $\mathrm{SC}^{4}(13 \mathrm{~b}, \mathrm{~d})$ mais paraît impossible dans le cas des CPA $(13 \mathrm{a}, \mathrm{c})$ :

(13a) *C'est la porte fermée que Marie s'en alla.

(13b) C'est après qu'elle a fermé la porte que Marie s'en est allée.

(13c) *C'est l'avion ayant atterri avec une heure de retard qu'elle a pris un taxi.

(13d) C'est parce que l'avion a atterri avec une heure de retard qu'elle a pris un taxi.

L'impossibilité de cliver la CPA comme on peut le faire pour n'importe quel composant de la prédication semble être un indice de la non-intégration de celle-ci dans la structure argumentale de la prédication régissante. Un deuxième test, celui de la nominalisation des deux constructions en tant que syntagme, tend à confirmer l'observation précédente $(14 \mathrm{a}-\mathrm{d})$ :

(14a) * La fermeture de la porte, Marie s'en est allée.

(14b) Après la fermeture de la porte, Marie s'en est allée.

(14c) *L'atterrissage de l'avion avec une heure de retard, elle a pris un taxi.

(14d) A cause de l'atterrissage de l'avion avec une heure de retard, elle a pris un taxi.

La nominalisation ne pose pas non plus de problèmes pour les $\mathrm{SC}(14 \mathrm{~b}, \mathrm{~d})$ mais n'est à nouveau pas envisageable pour les CPA $(14 \mathrm{a}, \mathrm{c})$. Les deux tests révèlent une opposition entre une construction syntaxiquement non intégrée dans la régissante, la $\mathrm{CPA}(1 \mathrm{~b}, 2 \mathrm{~b})$ et une construction intégrée à la structure argumentale de celle-ci, la SC (1b', 2b'). Ce constat peut sembler paradoxal dans la mesure où ces dernières contiennent un prédicat fini, alors que les premières proposent une forme verbale non finie. Cela tend à confirmer que la différence relevée ici n'est pas une différence de structure interne des prédications régies mais bien de rapport syntaxique réglant la dépendance entre les prédications.

Comment interpréter la différence des rapports syntaxiques entre prédications, observée dans les deux constructions? Nous basons notre analyse d'un point de vue théorique sur un classement des jonctions interprédicationnelle qui est proposé par la Role and Reference Grammar (RRG ; cf. Van Valin \& LaPolla 1997, Van Valin 2005, Buysse 2016) et qui est fondé sur une large gamme de langues typologiquement différentes.

\subsection{Le modèle de la RRG}

Au lieu d'adopter la dichotomie traditionnelle entre coordination et subordination ou la répartition classique entre classes (complétive, circonstancielle, ...) ou types (infinitives, participiales, ...) de subordonnées, la RRG fonde sa classification des liaisons de propositions sur le mode de jonction des prédications et sur le niveau de jonction de celles-ci.

3.2.1. Du point de vue du mode de jonction, la RRG distingue trois types de combinaisons sur la base de deux critères : la dépendance (dependency), la question de savoir si une des prédications combinées dépend syntaxiquement de l'autre, et l'intégration (embedding), c'est-à-dire le fonctionnement de la structure dépendante en tant que constituant ou adjoint au sein de l'autre (Van Valin \& LaPolla 1997).

(15a) Mon père est menuisier et ma mère est enseignante.

(15b) Il m'a dit qu'il ne viendra pas à la fête.

(15c) Il a arrêté de fumer des cigarettes. 
Toute forme de liaison qui n'implique ni dépendance ni intégration est considérée comme un cas de coordination (15a). La combinaison est alors caractérisée par les propriétés suivantes [- dépendance/ intégration]): chaque prédication impliquée dans la combinaison contient ses propres marqueurs grammaticaux et n'a pas besoin de l'autre pour fonctionner correctement. La subordination, par contre, est caractérisée par l'intégration d'une prédication dans l'autre et, en conséquence, par la dépendance de la première par rapport à la seconde Dans (15b), la prédication (qu')il ne viendra pas à la fête est intégrée comme objet direct dans la proposition matrice (Il me l'a dit) et, de ce fait, est dépendante de celle-ci. La combinaison présente alors les traits [+ intégration/ + dépendance]. La dépendance, dans ces cas-ci, est le produit de l'intégration syntaxique de la prédication dans la régissante. Enfin, si une prédication dépend bien d'une autre, mais sans y être intégrée en tant qu'adjoint ou constituant, la RRG parlera de cosubordination. Dans (15c), la prédication de fumer des cigarettes n'est pas intégrée dans la proposition matrice (cf. *Il l'a arrêté), mais dépend bien de celle-ci dans le sens où il ne porte pas de marqueurs de nombre, de personne ou de temps, ni n'est associé à un sujet particulier. Il y a donc une dépendance d'opérateurs (Van Valin \& LaPolla 1997), dans la mesure où la prédication dépendante a besoin de la matrice pour l'expression d'un ou plusieurs opérateurs grammaticaux ou d'un constituant essentiel, (le temps, le mode et le sujet). Bref, même si elle n'est pas intégrée fonctionnellement dans la régissante, la prédication régie ne peut pas fonctionner de manière indépendante [+ dépendance/ - intégration].

3.2.2. Les trois types de combinaisons peuvent être opérationnalisés à trois niveaux structurels de la phrase (les niveaux de jonction). Au niveau du noyau de la prédication ou niveau nucléaire (16a), la connexion de deux prédicats constituera un seul prédicat complexe avec un seul ensemble d'arguments ou de constituants partagés :

(16a) J'ai fait tomber le verre.

(16b) Fumer une cigarette tous les jours est très mauvais pour la santé.

(16c) Il passera dans l'après-midi parce qu'il a oublié hier soir son livre chez nous.

Au niveau cellulaire (16b), soit la cellule de la prédication régissante, la connexion se situe sur le plan des arguments du prédicat et implique plusieurs prédicats avec leurs propres arguments obligatoires. Enfin, au niveau de la clause (16c) la connexion se situe sur le plan des adjonctions périphériques de la prédication régissante. Deux clauses complètes sont combinées, incluant non seulement les arguments obligatoires, mais également d'autres éléments périphériques.

La combinaison des deux paramètres permet à la RRG de proposer une hiérarchie syntaxique des relations interpropositionnelles dans le cadre de laquelle (a) le niveau structurel le plus profond de la connexion (niveau nucléaire) entraînera toujours une jonction plus forte qu'un niveau structurel moins profond (niveau cellulaire ou clausal) et (b) une combinaison cosubordonnée impliquera toujours une jonction plus forte qu'une combinaison subordonnée ou coordonnée à un même niveau structurel.

(17a) Il était fatigué par ces déplacements et il est rentré se coucher.

(17b) Comme il était fatigué par ces déplacements, il est rentré se coucher.

(17c) Ces déplacements l'ayant fatigué, il est rentré se coucher.

Ainsi, au même niveau de jonction clausal (le niveau structurel le moins profond), la cosubordination (17c)) implique une jonction plus forte que la subordination) (17b), qui à son tour représente une jonction plus forte que la coordination (17a).

Le cadre d'appréhension de la RRG permet de comprendre le paradoxe, relevé par divers chercheurs, que la présence d'un connecteur interpropositionnel n'instaure pas nécessairement la jonction la plus étroite entre les prédications, dans la mesure où ces marqueurs permettent souvent de maintenir l'intégrité de la prédication dépendante et dès lors restreignent le niveau de dépendance de celle-ci, tandis que la dépendance d'opérateurs réduit l'intégrité de la prédication régie : "The more integrated the two events are, the less are they likely to be separated by a subordinator (or a physical pause) » (Givón, $1990: 560$ $561)$. 


\subsection{Caractérisation de la dépendance syntaxique des prédications régies}

La différence entre les CPA et les SC examinées ne s'explique pas par un niveau de jonction différent des deux constructions dans la mesure où les deux se situent à un niveau périphérique par rapport au noyau de la régissante. Cela se manifeste entre autres par les lectures circonstancielles dans les deux cas. Par contre, une différence nette apparaît quant au type de connexion convoqué.

La SC (1b', 2b'), comme les tests sous 3.1. le soulignent, est intégrée dans la régissante pour y remplir une fonction périphérique. De par son intégration, elle sera aussi hiérarchiquement dépendante de cette même prédication régissante et se situe donc dans un rapport de subordination. L'intégration est marquée par la présence d'un connecteur et la présence de ces deux caractéristiques (intégration + présence d'un connecteur) sont à leur tour à la base des contraintes interprétatives pesant sur les SC.

La CPA (1b, 2b), par contre, n'est pas fonctionnellement intégrée dans la régissante, tout en dépendant de cette dernière pour toute une série d'opérateurs TAM, étant donné le caractère non fini de son prédicat participial. Le rapport syntaxique entre les deux prédications consiste donc en une dépendance d'opérateurs sans intégration de la prédication régie comme argument ou adjonction pérphérique dans la prédication régissante, soit un rapport de co-subordination. Ce type de rapport implique l'absence d'intégration dans la structure syntaxique de la prédication régie et aussi l'absence d'un connecteur interpropositionnel. La présence de ces deux caractéristiques (non-intégration + absence de connecteur) explique la sousspécification des lectures des CPA.

\section{Conclusions}

Notre contribution permet donc de distinguer sur différents plans les propriétés syntaxo-sémantiques des CPA et des SC :

(1) le mode d'élaboration de l'interprétation circonstancielle est radicalement différent dans les SC et les CPA : il est plus contraint mais aussi plus précis dans le premier cas, moins contraint mais aussi sousspécifié dans le second cas. Par ailleurs, les interprétations circonstancielles ne sont pas les seules lectures générées par les CPA ;

(2) le mode de connexion entre les prédications est totalement différent : dans le cas de la SC, la connexion est réalisée explicitement et directement par l'insertion d'un élément spécifique, tandis que dans le cas de la CPA, la connexion est réalisée indirectement et implicitement par un manque, une absence affectant une des prédications et la rendant en conséquence dépendante de l'autre ;

(3) la force du lien syntaxique entre les prédications est différent, de type subordonnant dans le cas des SC, de type co-subordonnant dans le cas des CPA.

Les énoncés de type (1b', 2b') sont caractérisés par une intégration des prédications, explicitée par un marqueur, ce qui leur attribue un sens (relativement) spécifique. Par contre, les énoncés de type (1b, 2b) ne présentent pas d'intégration des prédications mais une dépendance d'opérateurs, qui n'est pas soulignée par un marqueur spécifique mais plutôt par une absence de marques. Cela explique leur lecture sous-spécifiée.

La contribution permet de fonder la distinction entre « circonstances » et « circonstancielle » (la classe de subordonnées) et de nourrir le débat à propos de la spécification/ sous-spécification des catégories et des fonctions en grammaire.

En morpho-syntaxe, il existe des catégories, des structures, voire des relations sous-spécifiées (cf. p.ex. Farrell, 2001), c'est-à-dire des composants de l'énoncé qui ne disposent pas d'une série d'informations déterminées, celles-ci étant alors suppléées par le contexte propositionnel ou discursif. Relevons en particulier, le phénomène de sous-spécification relationnelle entre deux énoncés propositionnels, qui est souvent rattaché à certains genres ou aux types de texte (cf. à ce propos Delbecque, 2006, : 242-246, qui reprend la liste des types de relations de cohérence de Mann \& Thompson, 1988). Ces configurations sousspécifiées sont particulièrement sensibles à la variation interprétative, comme c'est le cas des CPA que nous avons étudiées. 


\section{Références bibliographiques}

Arrivé, M., Gadet, F., et Galmiche, M. (1986). Le français d'aujourd'hui. Paris : Flammarion.

Buysse, M. (2016). L'acquisition de la liaison de propositions en L2 : Traitement et apport de la Role and Reference Grammar. Helsinki: Helsingin Yliopisto.

Cristofaro, S. (2003). Subordination. Oxford: Oxford University Press.

Dixon R. M. W. (2011). The Semantics of Clause Linking in Typological Perspective. In Aikhenvald, A. et Dixon, R. M. W. (éds), The Semantics of Clause Linking: a cross-linguistic typology. Oxford (United Kingdom): Oxford University Press, 1-55.

Delbecque, N. (2006). Linguistique cognitive. Comprendre comment fonctionne le langage. Louvain-la-Neuve : De Boeck Supérieur.

Farrell, P. (2001). Functional shift as category underspecification. English Language and Linguistics 5, 109-130.

Givón, T. (1990). Syntax. A functional-typological introduction, Volume II. Amsterdam \& Philadelphia: John Benjamins.

Goes, J. (2008). Les prédications secondes à prédicat adjectival. In Havu, E. et Pierrard, M. (éds), La prédication seconde : essai de mise au point. Travaux de linguistique 57, 23-41.

Grevisse, M. et Goosse, A. (15éd. 2011). Le bon usage. Grammaire française. Bruxelles : De Boek.

Hanon, S. (1989). Les constructions absolues en français contemporain. Louvain-Paris : Peeters.

Haspelmath, M. (1995). The converb as a cross-linguistically valid category. In Haspelmath M. et König, E. (éds), Converbs in Cross-Linguistic Perspective. Berlin-New York: Mouton de Gruyter, 1-55.

Havu, E. (2004). L’interprétation des constructions participiales appositives. La Linguistique 40, 65-82.

Havu, E. et Pierrard, M. (2015). Prédication seconde et (non-)marquage de la liaison. Langages 200, 15-30.

Havu, E. et Pierrard, M. (2016a). Constructions participiales absolues et liaisons de prédications : au-delà du lien syndétique. In Neveu, F. (éd.), Actes du $5^{e}$ Congrès Mondial de Linguistique Française (CMLF 16), SHS Web of Conferences 27, 14005, DOI: 10.1051/shsconf/20162714005.

Havu, E. et Pierrard, M. (2016b). A propos de la convergence entre adjoints adjectivaux et adverbiaux. In Spitzl-Dupic, F., Grégoire, M., Lebas-Fraczak, L., et Ryan, R. (éds.), Sur les traces de l'adjectif. Clermont-Ferrand: Presses universitaires Blaise-Pascal, 45-57.

Havu, E. et Pierrard, M. (2016c). Co-prédications adjectivantes : le degré de détermination du lien nominal entre les prédications. In Gautier, A., Havu, E., et Van Raemdonck, D. (éds.), DéterminationS. Brussels - Bern - Berlin: P.I.E.-Peter Lang, 27-45.

Hobaek Haff, M. H. (2010). A contrastive analysis of absolute constructions in French, German and Norwegian. Lingvistica investigationes, 208-224.

Himmelmann, Nikolaus P. et Schultze-Berndt, E. (2005). Issues in the syntax and semantics of participant-oriented adjuncts: an introduction. In Himmelmann, Nikolaus P. et Schultze-Berndt, E. (eds), Secondary Predication and Adverbial Modification. Oxford: Oxford University Press, 1-67.

Lehmann, C. (1989). Towards a typologie of clause linkage. In Haiman, J. et Thompson, S.A. (éds), Clause combining in grammar and discourse. Amsterdam \& New York: John Benjamins, 181-225.

Mann, W.C. et Thompson, S.A. (1988). Rhetorical Structure Theory: Toward a functional Theory of Text Organization. Text 8 (3), 243-281.

Mouret, F. (2011). Deux types de constructions absolues dans La Jalousie de Robbe-Grillet. L'information grammaticale, 51-56.

Muller, C. (2000). Les constructions à adjectif attribut de l'objet, entre prédication seconde et complémentation verbale. Langue française 127, 21-35.

Müller-Lancé, J. (1998). Die Verwendung absoluter Konstruktionen in französischen Printmedien. In Hellrich, U. et Klöden, H. (éds), Mediensprache in der Romania. Wilhelmsfeld: Gottfried Egert Verlag, 257-277. 
Riegel, M. (1991). Pour ou contre la notion grammaticale d'attribut de l'objet : critères et arguments. In de Gaulmyn, M.-M., Rémi-Giraud, S. (éds) A la recherche de l'attribut. Lyon : Presses Universitaires de Lyon, 99-118.

Riegel, M., Pellat, J.-C., et Rioul, R. (2009 [1994]). Grammaire méthodique du français. Paris : Presses universitaires de France.

Van Valin, R. D. Jr. et LaPolla, R. (1997). Syntax: Structure, Meaning and Function. Cambridge: Cambridge University Press.

Van Valin, R. D. Jr. (2005). Exploring the syntax-semantics interface. Cambridge: Cambridge University Press.

\footnotetext{
${ }^{1}$ Havu \& Pierrard (2016a) montrent néanmoins que la distinction entre les deux types n'est pas aussi radicale que Hanon (1989) et Mouret (2011) le postulent.

${ }^{2}$ Corpus incluant des textes écrits (journalistiques et littéraires) et oraux (journaux ou débats télévisés) et constitué par les chercheurs eux-mêmes et leurs étudiants en Master 2.

${ }^{3}$ Dans $7 \mathrm{~b}$ ' et $7 \mathrm{c}$ ', un participe passé serait toutefois impossible :

(7b') L'appartement nettoyé, ses parents arrivent (*Pendant que l'appartement est nettoyé, ses parents arrivent) (7c') *Ses parents arrivés bientôt, Paul nettoie l'appartement.

${ }^{4}$ Dans le cas de subordonnées en comme, puisque ou étant donné que, cela paraît beaucoup plus difficile, mais pas totalement impossible :

(a) Pour Henri Hatlan, toute hypothèse scientifique vraiment nouvelle et novatrice est de l'ordre du délire ou du fantasme. C'est étant donné que ce délire, ou ce fantasme, accepte de se modifier, ou alors d'être abandonné dans des confrontations, qu'il s'en écarte (www.sexopedie.com/fantasme.php)

(b) [...] le seul critère déterminant du domaine réservé est celui de l'engagement international. C'est puisque l'Etat s'est internationalement engagé que l'on parle du domaine. C'est puisque les Etats se sont fédérés à l'Etat fédéral qu'il y a répartition des compétences (https://www.memoireonline.com > ... > Droit International Public)

(c) C'est comme il a expliqué aux policiers que cela s'est passé.
} 